\title{
La radioadaptation : aspects cellulaires et moléculaires d'une réponse aux faibles doses de radiations ionisantes
}

\author{
O. RIGAUD *
}

(Manuscrit reçu le 6 juillet 1998, accepté le 8 juillet 1998)

RÉSUMÉ Il est bien établi que des doses sublétales d'agents endommageant l'ADN induisent des mécanismes de protection à un traitement ultérieur par de fortes doses; c'est le cas du phénomène de radioadaptation pour les radiations ionisantes. Depuis l'expérience princeps décrite en $\mathbf{1 9 8 4}$, de nombreux travaux ont confirmé la radioadaptation en terme de réduction de cassures chromosomiques pour divers modèles cellulaires in vitro ainsi qu'in vivo. De plus, l'adaptation contre l'induction de mutations géniques et la létalité est clairement démontrée. Cette revue tente de faire le point sur les résultats expérimentaux qui ont contribué à caractériser divers aspects de la radioadaptation. Le mécanisme moléculaire reste encore mal défini. L'expression de cette réponse nécessite une synthèse de novo de transcrits et de protéines dans l'intervalle de temps entre les deux doses. Des données sont en faveur des hypothèses selon lesquelles ces produits de gènes nouvellement synthétisés seraient impliqués dans l'activation de systèmes de réparation et des enzymes du métabolisme antioxydant. Une question majeure reste en suspens : celle de l'incidence du phénomène de radioadaptation sur l'évaluation du rique de cancer lié à l'exposition à de faibles doses, une préoccupation majeure en radioprotection. En l'absence de données expérimentales rigoureuses dans ce domaine, il parait erroné d'évoquer des effets bénéfiques de la radioadaptation.

ABSTRACT Radioadaptation: cellular and molecular features of a response to low levels of ionizing radiation

It is well established that sublethal doses of DNA damaging agents induce protective mechanisms against a subsequent high dose treatment; for instance, the phenomenon of radioadaptation in the case of ionizing radiations. Since the early observation described in 1984, numerous studies have confirmed the radiodaptive response in terms of reduction of chromosomal breaks for varied biological models in vitro and in vivo. Evidence for an adaptive response against the induction of gene mutations and the lethal effect is clearly demonstrated. This paper reviews the experimental results describing various aspects of these adaptive responses expressed on these different biological end-points. The molecular mechanism underlying radioadaptation still remains unclear. The development of this phenomenon requires $d e$ novo synthesis of transcripts and proteins during the time interval between the two doses. Some data are consistent with the hypotheses that these gene products would

Institut Curie-Recherche, UMR218 CNRS, LRC1 CEA, 26 rue d'Ulm, 75248 Paris cedex 05, France. Mél : odilc.rigaud@curie.fr 
be involved in the activation of DNA repair pathways and antioxidant systems. However, a major question still remains unanswered; indeed, it is not clear whether or not the radioadaptation could affect the estimation of cancer risk related with low level exposure to ionizing radiation, a major concern in radioprotection. Until such data are available, it is yet unwise to evoke the beneficial effects of radioadaptation.

\section{Introduction}

En réponse à une exposition à des stress génotoxiques générés par des agents physiques ou chimiques, l'activation des voies de transduction du signal déclenche divers processus moléculaires participant entre autres au contrôle de la progression du cycle cellulaire, à la réparation des lésions de l'ADN et à l'apoptose. De plus, de nombreuses études montrent à l'évidence que des doses sublétales d'agents endommageant l'ADN induisent des mécanismes de tolérance à un traitement ultérieur par de fortes doses. L'adaptation aux agents alkylants chez les bactéries en est le premier exemple décrit en 1977 (Samson et Cairns, 1977), une réponse est aussi mise en évidence peu après dans les cellules de mammifères (Samson et Schwartz, 1980). Dans le cas des radiations ionisantes (RI), la radioadaptation réfère au phénomène par lequel des cellules pré-exposées à une dose sublétale de quelques cGy dite dose adaptative deviennent moins sensibles aux effets génotoxiques d'une dose plus élevée de plusieurs Gy dite dose test délivrée quelques heures plus tard (UNSCEAR, 1994). L'existence de processus de tolérance inductibles par de faibles doses est également illustrée par les observations décrites sur la survie cellulaire après des doses uniques < $1 \mathrm{~Gy}$ : en effet, comparativement à la sensibilité attendue de l'extrapolation des effets des doses plus élevées, on constate une létalité par unité de dose plus forte pour des doses < 0,3Gy suivie d'une augmentation de radiorésistance dans la plage de doses intermédiaires 0,3-1Gy (Joiner et al., 1996).

À ce stade, on peut se demander ce qu'est une faible dose? D'un point de vue pragmatique, le terme «faible » dose définit toute dose pour laquelle un effet biologique ne peut être décelé en raison des limites de sensibilité des techniques actuellement disponibles. Des seuils de sensibilité de $2 \mathrm{cGy}$ pour la détection d'une augmentation de la fréquence d'aberrations chromosomiques et de $20 \mathrm{cGy}$ pour celle du risque de cancer ont été retenus au coût d'une analyse extensive de cas et ce par plusieurs équipes. Ainsi, pour évaluer les effets de doses dites faibles, il est admis d'extrapoler les effets établis pour des expositions à de fortes doses, et en particulier pour le risque de cancer. Cette pratique se fonde sur le concept selon lequel toute dose a un effet biologique même si celui-ci n'est pas décelable expérimentalement; on admet, par prudence, que cette extrapolation est linéaire quel que soit la dose, l'effet augmentant graduellement avec celle-ci. Ceci exclue l'existence d'un seuil de dose en-dessous duquel aucun effet n'est attendu ainsi que celle d'effets infralinéaires ou supralinéaires dus respectivement à l'activation ou à la non activation de processus moléculaires nécessaires à la gestion des dommages. 
Des phénomènes tels que la réponse adaptative et la radiorésistance induite suggèrent que la réponse biologique à une exposition aux faibles doses au niveau cellulaire est plus complexe que celle déduite de la simple extrapolation des effets des fortes doses. Cette revue tente de faire le point sur les données actuelles montrant l'existence de processus de tolérance déclenchés par de faibles doses de radiations et d'intégrer les connaissances acquises contribuant à la compréhension de leur mécanisme encore mal élucidé. L'essentiel de cette revue porte sur la radioadaptation et le lecteur peut se rapporter à des revues récentes sur la survie cellulaire après des doses uniques < 1Gy (Joiner et al., 1996 et références citées).

\section{La réponse radioadaptative chez les procaryotes et les eucaryotes inférieurs}

Chez les bactéries, il a été décrit différents processus inductibles en réponse à des agents endommageant l'ADN impliquant l'augmentation de l'activité des enzymes de la réparation (pour revue voir Walker, 1984). L'induction d'une radiorésistance consécutive à une pré-irradiation a été décrite chez Escherichia coli; cependant, les doses très élevées (>Do) laissaient supposer l'induction éventuelle du système SOS. Le système SOS permet une meilleure survie aux dommages mais ce au coût d'une mutagénèse élevée, un mécanisme différant donc de l'adaptation qui réduit à la fois la létalité et la mutagenèse. Un stress oxydant par le péroxyde d'hydrogène $\left(\mathrm{H}_{2} \mathrm{O}_{2}\right)$, générant des lésions via la production d'espèces radicalaires de l'oxygène du même type que celles induites par les RI, est capable d'induire une réponse adaptative chez les bactéries distincte de celle aux alkylants (Demple et Halkook, 1983). Cette réponse est caractérisée par l'induction de protéines sous le contrôle du régulon oxyR (Christman et al., 1985). De plus, on constate qu'un tel prétraitement déclenche une meilleure survie à une irradiation ultérieure par 10-150 Gy de rayons gamma (Demple et Halkook, 1983).

Chez la levure Saccharomyces cerevisiae, la réponse radioadaptative décrite dès 1984, se traduit par une radiorésistance proportionnelle à la dose adaptative et oxygène-dépendante ainsi que par une meilleure tolérance à l'effet mutagène (Mitchel et Morrison, 1984). Cette réponse non inductible chez les mutants rad52, défectifs dans la réparation des cassures double-brin, suggère que la réparation par recombinaison est impliquée. De plus, l'adaptation est croisée chez $S$. cerevisiae, c'est-à-dire que la pré-exposition à divers agents, des RI, des radiomimétiques, un choc thermique léger ou une privation en éléments nutritifs réduit la sensibilité au même agent ou à l'un quelconque de ces agents. Cette adaptation croisée suggère des étapes communes dans le mécanisme protecteur contre ces différents agents (Boreham et Mitchel, 1991).

\section{Première évidence de la réponse radioadaptative dans les cellules de mammifêres}

Dans l'expérience princeps décrite en 1984 par Olivieri et al. (1984), des lymphocytes humains stimulés étaient soumis à une exposition chronique aux rayonnements 
$\beta$ provenant de la thymidine tritiée $(370 \mathrm{~Bq} / \mathrm{ml})$ et irradiés en phase $\mathrm{G} 2$ par une dose de 1,5 Gy de rayons $X$ (Olivieri et al., 1984). Il en résultait une réduction de la fréquence d'aberrations chromosomiques en comparaison de celle radio-induite dans les lymphocytes non exposés au précurseur radioactif. Cette réduction observée indépendamment du temps de fixation après la dose-test suggère que la réponse n'est pas due à des perturbations du cycle associée à des différences de sensibilité cycle-dépendante. L'hypothèse d'une mort sélective d'une population plus radiosensible ayant incorporé la thymidine tritiée fut écartée ainsi que celle de facteur diffusible (Wiencke et al., 1986). Ultérieurement, il était montré qu'une dose aiguë très faible $(<1 \mathrm{cGy})$ est aussi efficace que l'exposition chronique et donc que la réponse n'est pas restreinte aux seules cellules en phase $S$, celles susceptibles d'incorporer le précurseur radioactif mais aussi inductible dans les cellules situées dans d'autres phases du cycle cellulaire (Shadley et al., 1987).

Depuis, de nombreux travaux ont confirmé l'existence du phénomène de radioadaptation à l'effet clastogène in vitro pour divers modèles cellulaires provenant de mammiferes ou d'autres espèces (poisson, insecte, plante...) ainsi qu' in vivo (UNSCEAR, 1994). Toutes ces études utilisant surtout le lymphocyte humain comme modèle, ont contribué à caractériser cette réponse qui se traduit aussi par une protection contre l'induction de mutations géniques et l'effet létal.

\section{Caractéristiques cellulaires de la réponse adaptative à l'effet clastogène}

La réduction de l'effet clastogène évalué par la fréquence d'aberrations chromosomiques ou de micronoyaux, est en moyenne de $50 \%$, comparé à l'effet de la seule forte dose.

Une faible dose est adaptative si elle est délivrée à un débit approprié à son intensité (Cai et Liu, 1990; Ikushima, 1989a; Shadley et Wiencke, 1989). En d'autres termes, il existe une fenêtre de doses pour lesquelles un certain nombre de signaux doivent être perçus par la cellule en un temps donné afin de déclencher le mécanisme responsable de la protection à l'exposition ultérieure. Le développement de la réponse adaptative nécessite un intervalle de temps minimum de 4 heures entre les deux doses (Ikushima, 1989a; Sasaki, 1995; Shadley et al., 1987).

La réponse est transitoire et persiste durant trois cycles; elle peut ensuite être réinduite (Cai et Liu, 1990; Shadley et al., 1987). C'est un phénomène de toutou-rien, il n'y a pas d'effet additif de deux doses faibles successives si la première déclenche la protection (Fan et al., 1990).

Comme il a été évoqué pour la levure, l'adaptation des cellules de mammiferres est croisée. La préexposition à divers agents dont $\mathrm{H}_{2} \mathrm{O}_{2}$, la bléomycine, la mitomycine $\mathrm{C}$, un choc thermique faible ou des enzymes de restriction générant des cassures 
double-brin (CDB) sont capables de déclencher la radioadaptation. De même, une faible dose de $\mathrm{RI}$ permet une meilleure tolérance à la bléomycine, $\mathrm{H}_{2} \mathrm{O}_{2}$ etc. (Cai et Jiang, 1995; Ikushima, 1989b; Wolff et al., 1988). Par ailleurs, des rayonnements de TEL élevés n'induisent pas la radioadaptation alors qu'une pré-irradiation de faible TEL permet une résistance à l'effet clastogène par le radon ou les neutrons (Ikushima, 1989b; Khandogina et al., 1991 ; Wolff et al., 1991). On constate donc une dépendance du type de lésions produites par les agents utilisés lors du prétraitement pour donner le signal d'induction.

La radioadaptation est un phénomène inductible in vivo chez l'animal préexposé à une dose unique de l'ordre du cGy ou soumis à une irradiation chronique à très faible débit. La réduction des cassures chromosomiques est mise en évidence dans les lymphocytes soumis à une exposition ultérieure in vitro (Cai et Liu, 1990; Cai et al., 1992; Wojcik et Tuschl, 1990). Dans le cas d'exposition aux deux irradiations adaptative et test in vivo, la réponse est observée au niveau des cellules somatiques et germinales (Cai et Liu, 1990). Chez l'homme, quelques études suggèrent l'existence d'une radioadaptation liée à une exposition occupationnelle ou accidentelle (Barquinero et al., 1995; Tedeschi et al., 1996). Dans l'analyse de la réponse de lymphocytes d'enfants vivant à Chernobyl au moment de l'accident, l'adaptation à l'effet clastogène paraît être corrélée à la contamination interne par le ${ }^{137} \mathrm{Cs}$ mais non à l'antécédent d'irradiation aiguë lors de l'accident.

Il est par ailleurs important de constater que la variabilité de la réponse de lymphocytes issus de donneurs normaux : pour certains individus, la réponse ne peut être induite et dans de rares cas, un effet synergique des doses adaptative et test est observé (Bosi et Olivieri, 1989; Shadley, 1994). Les raisons de cette variabilité inter-individuelle divergent selon les auteurs. Chez les non-répondeurs, l'adaptation peut être rétablie par l'addition de certains facteurs de croissance mettant ainsi en cause l'état physiologique des cellules au moment du prétraitement (Olivieri et Bosi, 1992). Certains auteurs évoquent que des modifications de la progression du cycle par la dose adaptative seraient à l'origine de cette variabilité inter-individuelle (Aghamohammadi et Savage, 1991 ; Wojcik et Streffer, 1995). Cette hypothèse est réfutée par d'autres (Dominguez et al., 1993; Salone et al., 1996; Wolff, 1996). En effet, Salone et al. (1996a) ont montré qu'une dose de 2 cGy peut provoquer des perturbations du cycle, une observation à souligner révélant l'effet biologique de faibles doses. Cependant, leur étude ultérieure incluant de donneurs répondeurs et non-répondeurs indique qu'il n'y a pas de corrélation entre d'éventuelles altérations du cycle et l'inductibilité de la réponse adaptative (Salone et al., 1996b). La possibilité que la variabilité inter-individuelle soit liée à des différences dans la constitution génétique reste posée (cf. paragraphe 8).

Dans ce contexte, l'analyse de cellules issues de patients atteints de syndromes radiosensibles ou de mutants déficients dans la réparation s'avèrerait particulièrement intéressante. Cependant, les quelques (rares) données publiées ne permettent pas de conclusion claire. L'induction de la radioadaptation à l'effet clastogène est 
décrite pour deux lignées lymphoblastoides issues d'un patient atteint d'ataxia telangectasia (AT) homozygote et du parent hétérozygote, alors que les lymphocytes de deux patients AT ne s'adaptent pas (Nemethova et al., 1995; Seong et al., 1995). L'absence de réponse adaptative est aussi notée dans les lymphocytes issus de cinq patients atteints du Syndrome de Down sur six testés (Khandogina et al., 1991). Il apparaît clairement que des analyses supplémentaires sont nécessaires pour conclure sur un lien éventuel entre radiosensibilité intrinsèque et inductibilité de la réponse.

\section{Radioadaptation à l'effet mutagène}

La réponse adaptative à l'effet mutagène a été établie dans divers modèles cellulaires in vitro avec comme gène cible, le locus HPRT (hypoxanthine phosphoribosyl transférase). Les conditions d'inductibilité et les caractéristiques de cette réponse sont similaires à celles de l'adaptation à l'effet clastogène : nature de l'agent inducteur, fenêtre de dose, aspect transitoire, nécessité d'un laps de temps entre les deux doses et d'une synthèse de nouvelles protéines et de transcrits avant la dose test, adaptation croisée (pour revue, Rigaud et Moustacchi, 1996). D'autre part, la diminution de la fréquence de mutations dans les cellules adaptées est caractérisée par un changement majeur du spectre moléculaire de mutations : les mutations de type délétion, classe prédominante de mutations induites par une irradiation aiguë, sont préférentiellement réduites dans les cellules adaptées (Rigaud et al., 1993; Zhou et al., 1994). De plus, la nature moléculaire des mutations ponctuelles induites par la forte dose ne differe pas selon que les cellules aient été ou non pré-exposées à une faible dose (Rigaud et al., 1995). En clair, la faible dose agirait préférentiellement sur des lésions pré-mutagènes conduisant à des délétions.

Étant donné que les mutations ainsi que les cassures chromosomiques jouent vraisemblablement un rôle dans l'initiation de la transformation maligne, on peut penser que la réponse adaptive qui réduit l'effet mutagène et clastogène pourrait modifier le risque de cancer. Une telle supposition est encore loin d'être étayée par des données expérimentales. Cependant, on peut citer quelques travaux relatifs à l'effet sur la transformation néoplasique de lignées cellulaires. Une réduction de la fréquence de transformation spontanée d'un facteur 2 à 3 après des irradiations aussi faibles que 1 cGy a été mise en évidence dans le cas de deux modèles différents, la lignée murine C3H10T31/2 et une lignée humaine hybride fibroblastesHela (Azzam et al., 1996; Redpath et Antoniono, 1998). Il a été aussi décrit une diminution de la fréquence de transformation néoplasique radio-induite à laquelle est associée une réduction de cassures chromosomiques dans les cellules murines C3H10T31/2 adaptées (Azzam et al., 1994) mais non dans une autre lignée murine m5S (Sasaki, 1995). Quant aux conséquences sur l'induction de tumeurs, une seule étude indique une augmentation du délai d'apparition et une incidence réduite de lymphomes thymiques chez la souris Swiss adaptée par une pré-dose de 1 cGy, comparé 


\section{LA RADIOADAPTATION}

à l'irradiation forte seule (Bhattacharjee, 1996). Davantage de données expérimentales dans ce domaine utilisant des modèles variés paraissent nécessaires pour conclure quant à la contribution de la réponse adaptative sur le risque de cancer lié à un antécédent d'irradiation par de faibles doses et plus particulièrement d'irradiation chronique à très faible débit, situation la plus fréquente des expositions occupationnelles.

\section{Radioadaptation à l'effet létal}

Une amélioration de la survie d'un facteur 2 a été décrite pour diverses lignées cellulaires soumises à des conditions d'adaptation variant de l'exposition unique, fractionnée ou chronique à très faible débit de dose (Azzam et al., 1992; Boothmann et al., 1996; Laval, 1988; Shadley et Dai, 1992). L'étude détaillée de Boothman et al. (1996) incluant neuf lignées normales ou tumorales humaines soumises à des pré-expositions répétées à des doses entre $0,01 \mathrm{cGy}$ et $50 \mathrm{cGy}$ révèle que seules deux lignées tumorales s'adaptent si les pré-expositions sont dans une fenêtre de doses $1-10 \mathrm{cGy}$. En analogie avec l'adaptation aux effets clastogène et mutagène, il existe une fenêtre de doses adaptatives, une synthèse de novo de messagers et de protéines avant la dose test est nécessaire et la réponse est inductible in vivo (Yozenawa et al., 1996).

On constate que l'expression simultanée d'une adaptation aux divers effets génotoxiques de la forte dose n'est pas toujours observée. Par exemple, l'adaptation de lymphoblastes humains à l'effet mutagène n'est pas corrélée avec une meilleure survie (Rigaud et al., 1993) alors qu'il l'est pour d'autres modèles cellulaires (Laval, 1988; Sasaki, 1995). Une même observation est faite en ce qui concerne effet létal et effet clastogène (Azzam et al., 1992; Shadley et Dai, 1992). Ceci pose la question d'une éventuelle différence de mécanismes d'induction d'une protection contre la production de mutations, celle d'aberrations chromosomiques ou la létalité.

\section{Lien entre radioadaptation et induction de radiorésistance après faible dose unique?}

Des améliorations récentes dans les techniques d'évaluation de la clonogénicité permettent des mesures fiables de la survie de cellules irradiées par de faibles doses et donc une analyse détaillée de la structure de la courbe de survie dans la région des doses < 1Gy (Marples et Joiner, 1993; Wouters et Skarsgard, 1994). Dans la plage des très faibles doses $(<0,3 \mathrm{~Gy})$, la pente de la courbe est plus raide que celle observée pour les fortes doses, c'est-à-dire qu'il y a une plus grande létalité par unité de dose (phase HRS pour «hyper radiosensitivity »). Aux doses intermédiaires (0,3-1Gy), un épaulement de la courbe reflète l'induction de radiorésistance (phase IRR pour " induced radioresistance "), comme si le taux de dommages ou 
de signaux généré par ces doses est alors suffisant pour déclencher la réparation des lésions. Les données groupées de deux équipes sur la réponse de dix lignées de cellules tumorales de radiosensibilité différentes suggèrent que le degré de radiosensibilité intrinsèque des cellules tumorales reflète leur capacité à activer un mécanisme de protection, illustrée par les phases HRS et IRR dans la courbe de survie (Joiner et al., 1996).

D'autre part, la préexposition des cellules à une faible dose quelques heures avant l'irradiation à dose unique élimine la phase d'hypersensibilité aux doses $<0,3$ Gy. Ce phénomène est transitoire et peut être aboli par la présence de cycloheximide (Marples et Joiner, 1993) suggèrant l'activation par la pré-exposition d'un mécanisme de radiorésistance analogue à celui de la réponse adaptative. Par contre, les conclusions d'une autre équipe menant des expériences similaires sur une autre lignée sont divergentes (Wouters et Skarsgard, 1997). Des recherches axées sur l'élucidation des mécanismes de ces deux phénomènes de tolérance sont déjà en cours (Joiner et al., 1996; Wouters et Skarsgard, 1997) et permettront de déterminer si les processus sont distincts ou non.

\section{Mécanismes moléculaires impliqués dans la radioadaptation}

Il est clairement établi qu'une synthèse de novo de transcrits et de protéines dans l'intervalle de temps entre les deux doses est nécessaire pour qu'il y ait adaptation (Wolff et al., 1989; Ikushima, 1992; Boothman et al., 1996). Les produits de ces gènes pourraient être impliqués dans l'activation par la dose adaptative de systèmes de réparation et/ou du métabolisme antioxydant.

L'hypothèse « réparation » fut émise en 1986 par Wiencke et al. qui montraient que la 3-aminobenzamide, un inhibiteur de la polyADP-ribosyl transférase qui est une enzyme de détection/signalisation des cassures de brins de l'ADN (pour revue, Le Rhun et al., 1998), bloque l'expression de la réponse adaptative à l'effet clastogène. On peut supposer que le processus moléculaire déclenché par la faible dose agit préférentiellement sur les lésions conduisant à des évènements de délétion (cassure chromosomique et mutation de délétion) dus vraisemblablement à des cassures double-brin (CDB). En effet, des tests biochimiques d'évaluation spécifique de ce type de dommages révèlent une réparation plus efficace des CDB dans les cellules adaptées (Zhou et al., 1992; Ikushima, 1996). Dans ce contexte, l'hypothèse d'une implication de la protéine kinase ADN-dépendante peut être évoquée étant donné son rôle dans la réparation des cassures double-brin. L'expression similaire des protéines DNA-PKcs, Ku80 et Ku70 du complexe de cette kinase observée dans les lymhoblastes AHH-1 adaptés ou non adaptés semble rejeter notre hypothèse (résultats non publiés).

D'autre part, la réparation par excision de bases semblerait être impliquée dans la radioadaptation. En effet, Le et al. (1998) ont développé une technique de détection de bases endommagées particulièrement sensible puisque capable de détecter 
une thymidine glycol ( $\mathrm{Tg}$ ) sur $10^{9}$ bases, (4,3 $\mathrm{Tg}$ sont décelées après $5 \mathrm{cGy}$ ); ils montrent que l'excision de ces bases endommagées est plus rapide dans les cellules adaptées par 0,25 Gy : $50 \%$ des Tg induites par la dose test de 2 Gy sont excisées en $50 \mathrm{~min}$ comparé à $100 \mathrm{~min}$ dans les cellules exposées à la seule dose-test.

D'autre part, il faut souligner l'importance dans la réponse aux radiations de l'activation des voies de transduction du signal qui déclenche une cascade séquentielle d'évènements moléculaires aboutissant entre autres à la réparation des lésions (pour revue, Maity et al., 1997). L'activation de la voie médiée par la protéine kinase $\mathrm{C}(\mathrm{PKC})$ semble essentielle à l'induction de la réponse adaptative. En effet, son expression est bloquée par des inhibiteurs de la PKC et ce dans divers modèles in vitro ou in vivo (Ikushima, 1992; Liu, 1992; Sasaki, 1995 ; Wojewodzka et al., 1997). Le promoteur de tumeur TPA, qui à faibles concentrations est activateur de la $\mathrm{PKC}$ peut mimer la réponse d'un pré-traitement par une dose adaptative de rayons X (Sasaki, 1995). De plus, il n'y a pas d'expression de la réponse adaptative si des agents bloquent la modulation de la concentration des ions $\mathrm{Ca}^{++}$ cytoplasmiques, un tel changement étant capable d'activer la PKC (Wojewodzka et al., 1994). La question de la cible et de la nature du signal donné par la faible dose, membranaire et/ou nucléaire reste posée.

L'hypothèse « métabolisme antioxydant » s'appuie sur le fait qu'une augmentation de l'activité des enzymes du métabolisme anti-oxydant déclenchée par la prédose contribuerait à l'élimination des dommages dus aux espèces radicalaires de l'oxygène générées par l'irradiation test. Plusieurs études décrivant une activité accrue de certains enzymes de détoxication, catalase, superoxyde dismutase, glutathion peroxydase associée à une adaptation induite in vitro et in vivo sont en faveur de cette hypothèse (Feinendegen et al. 1988; Laval, 1988; Zhang et al., 1998, Rigaud et al., résultats soumis).

Dans le but d'identifier les produits de gènes dont la synthèse de novo est nécessaire au développement de l'adaptation, une approche consiste à screener des gènes candidats choisis pour leur rôle-clé dans la réponse cellulaire aux radiations ionisantes. Des études suggèrent le rôle de certains gènes impliqués dans la réparation des dommages de l'ADN et dans le contrôle du cycle cellulaire. Ainsi, les transcrits GADD45, FAC (Fanconi Anemia C) et cyclines A et B sont induits après une exposition à faible débit de dose permettant une adaptation à la survie de fibroblastes humains (de Toledo et al., 1995). Par ailleurs, nos travaux révèlent que la protéine p53 ne contribuerait pas spécifiquement au mécanisme d'adaptation des lymphoblastes AHH-1 (Rigaud et Moustacchi, 1997). Dans l'étude de Boothman et al. (1996) évoquée plus haut, l'adaptation des deux lignées tumorales U1-Me1 et Hep2 en terme de survie est corrélée à un niveau d'expression constitutive élevée de cyclin D1 et de PCNA non observé pour les autres lignées testées qui ne s'adaptent pas. D'autre part, la pré-exposition induit une augmentation de la cyclin A et celle de deux transcrits XIP5 et XIP13 (XIP pour X-ray Induced Proteins, Boothman et al., 1993). Les auteurs suggèrent que l'amélioration de la survie des 
cellules adaptées serait due à l'activation d'un complexe multienzymatique de réparation impliquant les produits des gènes activés, XIP et la protéine PCNA. Cette étude souligne le rôle de certaines protéines d'expression constitutive élevée dans le développement de la réponse adaptative. Comme nous l'avons évoqué plus haut (cf. paragraphe 4), on peut se demander si de telles différences dans l'expression génique pourraient être à l'origine de l'absence d'inductibilité de la réponse adaptative chez certains individus ou certaines souches de rongeurs.

Une autre approche visant l'élucidation du mécanisme moléculaire consiste à rechercher des gènes différentiellement exprimés dans les cellules adaptées ou non par de faibles doses. Pour cela, diverses méthodes dont celle de la représentation différentielle des ARN messagers (differential display of mRNAs) ont permis d'identifier des changements d'expression de gènes associés à divers processus biologiques dont la réponse à des stress génotoxiques (Liang et Pardee, 1992). Ainsi, cette approche a déjà permis d'identifier l'activation de gènes codant pour des protéines de choc thermique lors de l'induction de radiorésistance (phase IRR après faibles doses uniques) (Joiner et al., 1996; Sadekova et al., 1997). Des efforts ciblés dans cet axe de recherche devraient contribuer à la compréhension du mécanisme moléculaire de la radioadaptation.

\section{Conclusion}

L'ensemble de ces études indiquent clairement que l'exposition à de faibles doses déclenche des mécanisme protecteurs permettant une sensibilité réduite à l'induction des cassures chromosomiques, des mutations et de la létalité.

Les phénomènes de radioadaptation et de radiorésistance induite après une dose unique révèlent que des doses dites faibles sont capables de moduler les effets d'exposition à de fortes doses létales. Cette acquisition de tolérance seraient due à l'activation de systèmes de réparation par excision ou de celle des cassures double-brin et aussi à l'augmentation du potentiel antioxydant endogène. L'identification des produits de gènes nécessaires au développement de ces processus de tolérance permettront d'approfondir les connaissances sur le mécanisme moléculaire encore mal connu.

Une question majeure reste en suspens : dans quelle mesure, la radioadaptation, un phénomène bien établi dans des systèmes cellulaires peut affecter le risque de cancer associé à des expositions à de faibles doses? En l'absence de données à ce jour, il est prématuré d'évoquer des effets bénéfiques de l'adaptation. Il paraît essentiel de développer des recherches visant à définir l'incidence potentielle du phénomène de radioadaptation sur l'évaluation du rique de cancer lié à des irradiations faibles ou des expositions chroniques à bas débit, une préoccupation majeure en radioprotection. 


\section{LA RADIOADAPTATION}

Remerciements : Je remercie le Dr Ethel Moustacchi pour ses remarques particulièrement utiles sur le manuscrit. Le laboratoire UMR 218 du CNRS a reçu le soutien de l'ARC, de la Ligue contre le Cancer et du programme « Radiobiologie et Génotoxicologie » de l'Institut Curie.

\section{Références}

Aghamohammadi S.Z., Savage J.R.K. (1991) A BrdU pulse double-labelling method for studying adaptive response. Mutation Res., 251, 133-141.

Azzam E.I., de Toledo S.M., Raaphorst G.P., Mitchel R.E.J. (1992) Radiation-induced radioresistance in a normal human skin fibroblast cell line. In: Low dose irradiation and biological defense mechanisms (T. Sugahara, L.A. Sagan, T. Aoyama, Eds.) pp. 291-294. Elsevier Science, Amsterdam.

Azzam E.I., Raaphorst G.P., Mitchel R.E.J. (1994) Radiation-induced adaptive response for protection against micronucleus formation and neoplastic transformation in C3H 10T1/2 mouse embryo cells. Radiation Res., 138, S28-S31.

Azzam E.I., de Toledo S.M., Raaphorst G.P., Mitchel R.E.J. (1996) Low-dose ionizing radiation decreases the frequency of neoplastic transformation to a level below the spontaneous rate in $\mathrm{C} 3 \mathrm{H} 10 \mathrm{TI} / 2$ cells. Radiation Res., 146, 369-373.

Barquinero J.F., Barrios L., Caballin M.R., Miro R., Ribas M., Subias A., Egozcue J. (1995) Occupational exposure to radiation induces an adaptive response in human lymphocytes. Int. J. Radiat. Biol., 67, 187-191.

Bhattacharjee D. (1996) Role of radioadaptation on radiation-induced thymic lymphoma. Mutation Res., 358, 223-230.

Boothman D.A., Meyers, Fukunaka, M. Lees S.W. (1993) Isolation of X-ray-inducible transcripts in radioresistant melanoma cells. Proc. Nat. Acad. Sci. (USA), 90, 7200-7204.

Boothman D.A., Meyers M., Odergaard E., Wang M. (1996) Altered G1 checkpoint control determines adaptive survival response to ionizing radiation. Mutation Research, 358, 143-154.

Boreham D.R., Mitchel R.E.J. (1991) DNA lesions that signal the induction of radioresistance and DNA repair in yeast. Radiation Res., 128, 19-28.

Bosi A., Olivieri G. (1989). Variability of the adaptive response to ionizing radiations in humans. Mutation Res., 211, 13-17.

Cai L., Liu S.Z. (1990) Induction of cytogenetic adaptive response of somatic and germ cells in vivo and in vitro by low-dose X-irradiation. Int. J. Radiat. Biol., 58, 187-194.

Cai L., Jiang J. (1995) Mild hyperthermia can induce adaptation to cytogenetic damage caused by subsequent $\mathrm{X}$ irradiation. Radiation Res., 143, 26-33. 


\section{O. RIGAUD}

Christman M.F., Morgan R.W., Jacobson F.S., Ames B.N. (1985) Positive control of a regulon for defenses against some oxidative stress and some heat-shock proteins in Salmonella typhimurium. Cell, 41, 753-762.

Demple B., Halbrook J. (1983) Inducible repair of oxidative DNA damage in Escherichia coli. Nature, 304, 466-468.

Dominguez I., Panneerselvan N., Escalza P., Natarajan A.T., Cortés F. (1993) Adaptive response to radiation damage in human lymphocytes conditioned with hydrogen peroxide as measured by the cytokinesis-block micronucleus technique. Mutation Res., 301, 135-141.

Fan S., Vijayalaxmi, Mindek G., Burkart W. (1990) Adaptive response to 2 low doses of X-rays in human blood lymphocytes. Mutation Res., 243, 53-56.

Feinendegen L.E., Bond V.P., Booz J., Mühlensiepen H. (1988) Biochemical and cellular mechanisms of low-dose effects. Int. J. Radiat. Biol., 53, 23-37.

Ikushima T. (1987) Chromosomal responses to ionising radiation reminiscent of an adaptive response in cultured Chinese hamster cells. Mutation Res., 180, 215-221.

Ikushima T. (1989a) Radioadaptive response: Characterization of a cytogenetic repair induced by low level radiation in cultured chinese hamster cells. Mutation. Res., 227, 241-246.

Ikushima T. (1989b) Radio-adaptive response: a novel chromosomal response in Chinese hamster cells in vitro. In: Chromosomal aberrations, Basic and applied aspects (G. Obe, A.T. Natarajan, Eds.), pp. 151-162, Springer-Verlag, Berlin.

Ikushima, T. (1992) Radio-adaptive response: Involvement of induction of specific genes by low doses of ionising radiation. In: Low dose irradiation and biological defense mechanisms (T. Sugahara, L.A. Sagan, T. Aoyama, Eds.) pp. 255-258. Elsevier Science, Amsterdam.

Ikushima T. (1996) Radioadaptive response: Efficient repair of radiation-induced damage in adapted cells. Mutation Res., 358, 193-198.

Joiner M.C., Lambin P., Malaise E.P., Robson T., Arrand J.E., Skov, K.A. Marples B. (1996) Hypersensitivity to very-low single radiation doses: its relationship to radioadaptive response and induced resistance. Mutation Res., 358, 171-183.

Khandogina E.K., Mutovin G.R., Zvereva S.V., Antipov A.V., Zverev D.O., Akifyev A.P. (1991) Adaptive response in irradiated human lymphocytes: radiobiological and genetical aspects. Mutation Res., 251, $181-186$.

Laval F. (1988) Pretreatment with oxygen species increases the resistance of mammalian cells to hydrogen peroxide and g-rays. Mutation. Res., 201, 73-79.

Le X.C., Xing J.Z., Lee J., Leadon S.A., Weinfield M. (1998) Inducible repair of thymine glycol detected by an ultrasensitive assay for DNA damage. Science, 280, 1066-1069. 


\section{LA RADIOADAPTATION}

Le Rhun Y., Kirkland J.B., Shah G.M. (1998) Cellular responses to DNA damage in the absence of poly(ADP-ribose) polymerase. Biochem. Biophys. Res. Commun., 245, 1-10.

Liang P., Pardee A.B. (1992) Differential display of eukaryotic messenger RNA by means of the polymerase chain reaction. Science, 257, 967-971.

Liu S.Z. (1992) Multilevel mechanisms of stimulatory effect of low dose radiation on immunity. In: Low dose irradiation and biological defense mechanisms (T. Sugahara, L.A. Sagan, T. Aoyama, Eds.) pp. 225-232. Elsevier Science, Amsterdam.

Liu S.Z., Cai L., Sun S.Q. (1992) Induction of a cytogenetic adaptive response by exposure of rabbits to very low dose-rate $\gamma$-radiation. Int. J. Radiat. Biol., 62, 187-190.

Maity A., Kao, G.D., Muschel R., Mc Kenna W.G. (1997) Potential molecular targets for manipulating the radiation response. Int. J. Radiation Oncology Biol. Phys., 37, 639-653.

Marples B., Joiner M.C. (1993) The response of Chinese hamster V79 cells to low radiation doses: Evidence of enhanced sensitivity of the whole cell population. Radiation Res., 133, 41-51.

Marples B., Joiner M.C. (1995) The elimination of low-dose hypersensitivity in Chinese hamster V79379A cells by pretreatment with $X$ rays or hydrogen peroxide. Radiation Res., 141, 160-169.

Mitchel R.E.J., Morrison D.P. (1984) An oxygen effect for gamma-radiation induction of radiation resistance in yeast. Radiation Res., 100, 205-210.

Nemethova G., Kalina I., Racekova N. (1995) The adaptive response of peripheral blood lymphocytes to low doses of mutagenic agents in patients with ataxia telangiectasia. Mutation Res., 348, 101-104.

Olivieri G., Bodycote J., Wolff S. (1984) Adaptive response of human lymphocytes to low concentration of radioactive thymidine. Science, 223, 594-597.

Olivieri G., Bosi A. (1992) Possible causes of variability of the adaptive response in human lymphocytes. In: Chromosomal aberrations, Basic and applied aspects (Obe G., Natarajan A. T., Eds.) pp. 130-139. Springer-Verlag, Berlin.

Redpath J.L., Antoniono R.J. (1998) Induction of an adaptive response against spontaneous neoplastic transformation in vitro by low-dose gamme radiation. Radiation Res., 149, 517-520.

Rigaud O., Papadopoulo D., Moustacchi E. (1993) Decreased deletion-type mutation in radiodapted human cells. Radiation Res., 133, 94-101.

Rigaud O., Laquerbe A., Moustacchi E. (1995) DNA sequence analysis of HPRT mutants induced in human lymphoblastoid cells adapted to ionising radiation. Radiation Res., 144, 181-189.

Rigaud O., Moustacchi E. (1996) Radioadaptation for gene mutation and the possible molecular mechanisms of the adaptive response. Mutation Res. 358, 127-134. 


\section{O. RIGAUD}

Rigaud O., Moustacchi E. (1997) p53 activation in radioadapted human lymphoblastoid cells. Radioprotection, 32, C1-411-412.

Sadekova S., Lehnert S., Chow T.Y.K. (1997) Induction of PBP74/mortalin/Grp75, a member of the hsp 70 family, by low doses of ionizing radiation:a possible role in induced radioresistance. Int. J. Radiat. Biol., 72, 653-660.

Salone B., Grillo R., Aillaud M., Bosi A., Olivieri G. (1996a) Effects of low-dose (2 cGy) X-ray on cell-cycle kinetics and on induced mitotic delay in human lymphocyte. Mutation Res., 351, 193197.

Salone B., Pretazzoli V., Bosi A., Olivieri G. (1996b) Interaction of low dose irradiation and subsequent mutagenic treatment: role of mitotic delay, Mutation Res., 358, 155-160.

Samson L., Cairns J. (1977) A new pathway for DNA repair in Escherichia coli. Nature, 267, 281-283.

Samson L., Schwartz J.L. (1980) Evidence for an adaptive DNA repair pathway in CHO and human skin fibroblast cell lines. Nature, 287, 861-863.

Sasaki M.S. (1995) On the reaction kinetics of the radioadaptive response in cultured mouse cells. Int. J. Radiat. Biol., 68, 281-291.

Seong J., Suh C.O., Kim G.E. (1995) Adaptive response to ionizing radiation induced by low doses of gamma rays in human cell lines. Int. J. Radiat. Oncol. Biol. Phys., 33, 869-874.

Shadley J.D., Afzal V., Wolff S. (1987) Characterization of the adaptive response to ionizing radiation induced by low doses of X-rays to human lymphocytes. Radiation Res., 111, 511-517.

Shadley J.D., Wiencke J.K. (1989) Induction of the adaptive response by X-rays is dependant on radiation intensity. Int. J. Radiat. Biol., 56, 107-118.

Shadley J.D., Dai G. (1992) Cytogenetic and survival adaptive responses in G1 phase human lymphocytes. Mutation. Res, , 265, 273-281.

Shadley J.D. (1994) Chromosomal adaptive response in human lymphocytes. Radiation Res., 138, S9-S12.

Tedeschi B., Caporossi D., Vernole P., Padovani L., Mauro F. (1996) Do human lymphocytes exposed to the fallout of the Chernobyl accident exhibit an adaptive response? III. Challenge with bleomycin in lymphocytes from children hit by the initial acute dose of ionizing radiation. Mutation Res., 354, 77-80.

de Toledo S.M., Azzam E.I., Gasmann M.K., Mitchel R.E.J. (1995) Use of semiquantitative reverse transcription-polymerase chain reaction to study gene expression in normal skin fibroblasts following low dose-rate irradiation. Int. J. Radiat. Biol., 67, 135-143.

Ueno A.M., Vannais D.B., Gustafson D.L., Wong J.C., Waldren C.A. (1996) Alow adaptive of gammarays reduced the number and altered the spetrum of S1 mutants in human-hamster hybrid AL cell line. Mutation Res., 358, 161-170. 


\section{LA RADIOADAPTATION}

UNSCEAR report (1994) Adaptive responses to radiation in cell and organisms. In: Report to the General Assembly, scientific annex $B$ (united Nations sales publication EXI.11).pp. 185-272. United Nations, New York.

Walker G.C. (1984) Mutagenesis and inducible response to deoxyribonucleic acid damage in Escherichia coli. Microbiol. Review, 48, 60-93.

Wiencke J.K., Afzal V., Olivieri G., Wolff S. (1986) Evidence that the ${ }^{3} \mathrm{H}$-thymidine -induced adaptive response of human lymphocytes to subsequent dose of X-rays involves the induction of a DNA repair mechanism. Mutagenesis, 1, 375-380.

Wojcik A., Tuschl H. (1990) Indications of an adaptive response in C57BL mice pre-exposed in vivo to low doses of ionizing radiation. Mutation Res., 243, 67-73.

Wojcik A., Bonk K., Müller W.U., Streffer C., Weissenborn U., Obe G. (1992) Absence of adaptive response to low doses of $\mathrm{X}$-rays in preimplantation embryos and spleen lymphocytes of an inbred mouse strain as compared to human peripheral lymphocytes: a cytogenetic study. Int. J. Radiat. Biol., 62, 177-186.

Wojcik A., Streffer C. (1995) Application of a multiple fixation regimen to study the adaptive response to ionizing radiation in lymphocytes of two human donors. Mutation Res., 326, 109-116.

Wojewodzka M., Walicka M., Sochanowicz B., Szumiel I. (1994) Calcium antagonist, TMB-8, prevents the induction of adaptive response by hydrogen peroxide or $\mathrm{X}$-rays in human lymphocytes. Int. J. Radiat. Biol., 66, 99-109.

Wojewodzka M., Krusweski M., Szumiel I. (1997) Effects of signal transduction inhibition in adapted lymphocytes: micronuclei frequency and DNA repair. Int. J. Radiat. Biol., 71, 245-252.

Wolff S., Afzal V., Wiencke J.K., Olivieri G., Michael A. (1988) Human lymphocytes exposed to low doses of ionizing radiations become refractory to high doses of radiation as well as to chemical mutagens that induce double-strand breaks in DNA. Int. J. Radiat. Biol., 53, 39-48.

Wolff S., Wiencke J.K., Afzal V., Youngblom J., Cortes F. (1989) The adaptive response of human lymphocytes to very low doses of ionizing radiation: a case of induced chromosomal repair with the induction of specific proteins. In: Low Dose Radiation, Biological Bases of Risk Assesment (Baverstock K. F. and Stather J. W., Eds) pp. 446-454. Taylor and Francis, London.

Wolff S., Jostes R., Cross F.T., Hui T.E., Afzal V., Wiencke J.K. (1991) Adaptive response of human lymphocytes for the repair of radon-induced chromosomal damage. Mutation Res., 250, 299306.

Wolff S. (1996) Aspects of the adaptive response to very low doses of radiation and other agents. Mutation Res., 358, 135-142.

Wouters B.G., Skarsgard L.D. (1994) The response of a human tumor cell line: evidence of enhanced sensitivity. Radiation Res., 138, S176-S180. 


\section{O. RIGAUD}

Wouters B.G., Skarsgard L.D. (1997) Low-dose radiation sensitivity and induced radioresistance to cell killing in HT-29 cells is distinct from the «adaptive response » and cannot be explained by a subpopulation of sensitive cells. Radiation Res., 148, 435-442.

Yozenawa M., Misonoh J. Hosokawa Y. (1996) Two types of X-ray-induced radioresistance in mice: presence of 4 dose ranges with different biological effects. Mutation Res., 358, 237-243.

Zhang H., Zheng R.L., Wei Z.Q., Li W.J., Gao Q.X., Chen W.Q., Wang Z.H., He J., Liang J.P., Han G.W., Huang T., Li Q., Xie H.M., Zhang S.M., Cai X.C. (1998) Effects of pre-exposure of mouse testis with low-dose ${ }^{16} \mathrm{O}^{8+}$ ions or ${ }^{60} \mathrm{Co} \gamma$-rays on sperm shape abnormalities, lipid peroxidation and superoxide dismutase (SOD) activity induced by subsequent high dose irradiation. Int. J. Rad. Biol., 73, 163-167.

Zhou P.K., Sun W.Z., Liu X.Y., ZhangY.P., Wei K. (1992) Adaptive response of mutagenesis and DNA double-strand break repair in mouse cells induced by low dose of $\gamma$-ray. In: Low dose irradiation and biological defense mechanisms (Suguhara T., Sagan L. A., Aoyama T., Eds) pp. 271274. Elsevier Science, Amsterdam.

Zhou P.K., Zhiang X.Q., Sun W.Z., Liu X.Y, Zhang Y.P., Wei K. (1994) Adaptive response to mutagenesis and its molecular basis in a human T-cell leukemia line primed with a low dose of gamma rays. Radiat. Env. Biophys, 33, 211-217. 